\title{
Versorgungsprozesse und das Zusammenspiel der Sektoren im internationalen Vergleich
}

Verena Struckmann, Juliane Winkelmann und Reinhard Busse

Inhaltsverzeichnis

1.1 Hintergrund -5

$1.2 \quad$ Methode - 7

1.2.1 Länderauswahl - 7

1.2.2 Definitionen -7

1.3 Verschiebungen von Zuständigkeit, Organisation und Versorgungsprozessen - 10

1.3.1 Reformen in europäischen Ländern - 10

1.3.2 Tagesfälle und ambulante Operationen in Europa - 12

1.4 Neue Formen und Prozesse der Zusammenarbeit im ambulanten und stationären Sektor: Leistungserbringung und Patientenpfade in europäischen Ländern - 13

1.5 Zentrale Bereiche zur Überwindung von Schnittstellenproblemen - 18

1.5.1 Lösungsansätze für den Bereich Steuerung und Zuständigkeiten aus Finnland, den Niederlanden und Norwegen - 18

1.5.2 Lösungsansatz für den Bereich Finanzierung aus den Niederlanden - 19 
1.5.3 Lösungsansätze für den Bereich Digitalisierung aus Estland, Dänemark und den Niederlanden - 20

$1.6 \quad$ Zusammenfassung und Fazit - 21

Literatur - 22 


\section{- Zusammenfassung}

Eine sektorenübergreifende Sichtweise ist insbesondere vor dem Hintergrund der zahlreichen technischen Fortschritte, steigender Kosten und einer alternden Gesellschaft mit zunehmend chronischen, aber häufig ambulant behandelbaren Erkrankungen wichtig. Mehrere europäische Länder begegnen diesen Entwicklungen, indem sie die Leistungserbringung über die Sektorengrenzen hinweg neu gestalten und Versorgungsprozesse systematisch durch eine Re-Organisation der Gesundheitsversorgung im ambulanten und stationären Sektor steuern. Anhand der Beschreibung aktueller Reformen, Verschiebungen von Zuständigkeiten, dem Aufbau der Leistungserbringung anhand von Patientenpfaden mit besonderem Fokus auf Schnittstellen, Organisation und Kommunikation wird deutlich, dass diese Herausforderungen in anderen europäischen Ländern (Dänemark, Finnland, Niederlande, Norwegen und Österreich) bereits konkreter als in Deutschland angegangen wurden. Auch wenn sich Gesundheitssysteme unterscheiden, was das Lernen von anderen verkompliziert, gibt es durchaus Ansätze und dafür notwendige Voraussetzungen, die auch für den deutschen Kontext Anregungen bieten können.

A cross-sectoral approach is particularly important in the light of the many technological advances, rising costs and an ageing society with an increasing number of chronically ill people who can also be treated in ambulatory settings. Several European countries are countering these developments by reorganising the provision of services across sectoral boundaries and systematically managing care processes by re-organising health care in the outpatient and inpatient sector. Based on the description of current reforms, shifts of responsibilities, service provision with patient pathways across sectors, organisation and communication in several European countries (Austria, Denmark, Finland, the Netherlands and Norway), this chapter shows that compared to
Germany, these challenges have already been tackled more explicitly in the countries investigated. Even if health care systems differ, which complicates mutual learning, there are certainly approaches and necessary prerequisites that can also provide inspiration and ideas for the reorganisation of health service provision in Germany.

\subsection{Hintergrund}

Im Sinne einer kontinuierlichen, koordinations- und patientenorientierten Versorgung sind sektorenübergreifende Versorgungsstrukturen zentral. Das deutsche Gesundheitssystem ist aufgrund zahlreicher Kostenträger und Leistungserbringer allerdings stark von einer Fragmentierung der medizinischen Leistungen und Zuständigkeiten zwischen der ambulanten und stationären Versorgung geprägt. Diese Fragmentierung führt zu vielfachen Schnittstellen zwischen Personen, Versorgungseinrichtungen und -ebenen (Prävention, ambulante und stationäre Versorgung sowie Rehabilitation und Pflege), Sektoren oder Arbeitsprozessen, die unzureichend koordiniert sind. Diese Schnittstellen stellen einen neuralgischen Punkt dar und führen daher regelmäßig zu Informations-, Wirkungs- und Qualitätsverlusten, zu Doppeluntersuchungen und zu Einbußen bei der Wirtschaftlichkeit und behindern so eine bedarfsgerechte und kontinuierliche Leistungserbringung (Straub et al. 2016; European Union 2017; FES 2017). Darüber hinaus kann der medizinisch-technische Fortschritt als wesentlicher Kostentreiber identifiziert werden. Oftmals orientiert sich die Versorgung nicht am medizinischen oder Patientenbedarf, sondern an Regelungen zur Vergütung und Leistungserbringung, die für den jeweiligen Sektor spezifisch sind. Das gilt besonders für die Schnittstelle zwischen akutstationärer und ambulanter Versorgung, allerdings entstehen auch Brüche zwischen allgemein- und fachärztlicher Versorgung. Mit der immer älter werdenden Bevölkerung entstehen an diesen 
Schnittstellen zusätzliche neue Probleme und Herausforderungen, da beispielsweise Patienten den akutstationären Sektor zunehmend mit einem höheren Versorgungsbedarf aufgrund von chronischen Vorerkrankungen oder Multimorbidität verlassen (SVR 2012; Albrecht et al. 2020).

Die Verpflichtung zur Vermeidung von Schnittstellenproblemen wurde bereits im Jahr 2007 mit dem Gesetz zur Stärkung des Wettbewerbs in der gesetzlichen Krankenversicherung (GKV-WSG) festgehalten und im Rahmen des Gesetzes zur Verbesserung der Versorgungsstrukturen in der gesetzlichen Krankenversicherung (GKV-VStG) im Jahr 2011 noch einmal präzisiert (SVR 2012). Mit diesen Reforminitiativen, aber auch bereits vorher und seitdem wurde versucht, die mit den Sektorengrenzen verbundenen Defizite in der Versorgung zu beheben (z. B. mit der Einführung von Modellvorhaben nach §§ 63-65 SGB V, des Innovationsfonds nach § 92a SGB V, von MVZs nach § 95 (1a) SGB V, vor- und nachstationärer Behandlung nach $\S 115 \mathrm{a}$, ambulantem Operieren nach $\S 115 \mathrm{~b}$, der ambulanten spezialfachärztlichen Versorgung nach $\S 116 \mathrm{~b}$, der besonderen Versorgung nach $\S 140 a)$. Das Ergebnis ist ein inkonsistentes Sammelsurium verschiedener Einzelregelungen, deren bisherige Entwicklung deutlich hinter ihrem vermuteten Potenzial und den Erwartungen des Gesetzgebers, der beteiligten Akteure und den Anforderungen an eine bedarfsorientierte $\mathrm{Ge}-$ sundheitsversorgung blieb (Leber und Wasem 2016). Es fehlt damit weiterhin eine eigenständige kohärente gesetzliche Grundlage für eine Versorgungsform, die eine sektorenübergreifende Koordination als konstitutives Element bzw. als notwendige Bedingung voraussetzt. Die grundlegenden, historisch gewachsenen strukturellen Sektorengrenzen und die damit einhergehende Fragmentierung konnten bisher nicht bzw. nur unzureichend überwunden werden. Nicht ohne Grund halten wichtige Akteure (GKV-Spitzenverband, SVR) seit Jahren eine Reform des ambulant-stationären Schnittstellenbereichs für dringend notwendig (SVR 2009, 2012, 2018; GKV-SV 2017).

Auch andere europäische Länder stehen vor der Herausforderung, die Leistungserbringung über die Sektorengrenzen hinweg neu $\mathrm{zu}$ gestalten und Versorgungsprozesse systematisch zu steuern, um dadurch den Auswirkungen einer alternden Bevölkerung und einer steigenden Zahl von Menschen mit chronischen Erkrankungen wirksam zu begegnen. Ziel ist es, die Qualität und Effizienz der Gesundheitsversorgung zu steigern, Kosten einzudämmen und somit die Kosten-Effektivität zu erhöhen. So werden in einigen europäischen Ländern durch eine Re-Organisation der Gesundheitsversorgung verstärkt Zuständigkeiten verschoben, sodass ambulante Leistungen eine stationäre Versorgung ersetzen. Um die deutsche Versorgungssituation und das $\mathrm{Zu}-$ sammenspiel der Sektoren besser einordnen zu können, kann ein Blick in andere europäische Länder helfen, Strukturen und bereits etablierte Ansätze zu identifizieren, die eine erfolgreiche Implementierung in Deutschland erleichtern würden.

Dieser Beitrag untersucht, wie die Behandlungspfade zwischen den unterschiedlichen Sektoren und Versorgungsebenen in anderen europäischen Ländern organisiert und aufgeteilt sind, wie Arbeitsabläufe und Kommunikation zwischen ambulanten und stationären Leistungserbringern gestaltet und wie Schnittstellen organisiert sind. Anhand ausgewählter Länderbeispiele werden aktuelle Entwicklungen und Reformen in der Verschiebung von Zuständigkeiten, Organisation und Versorgungsprozessen aufgezeigt, die darauf zielen, die ambulante und stationäre Versorgung neu zu strukturieren und stärker zu integrieren. Anschließend wird anhand von Patientenpfaden dargestellt, wie unterschiedlich Versorgungsketten aufgebaut sind und wie die intersektorale Organisation und Kommunikation stattfindet. 


\subsection{Methode}

\subsubsection{Länderauswahl}

Auf Basis einer gezielten Literaturrecherche, der internationalen Gesundheitssystemprofil-Serie (Health Systems in Transition HiT) und länderspezifischen Gesundheitsprofilen des European Observatory on Health Systems and Policies sowie grauer Literatur wurden europäische Länder mit interessanten Ansätzen für die Neuorganisation und Koordination der Versorgung zwischen den verschiedenen Sektoren und Versorgungsebenen identifiziert. Dabei bestand ein besonderes Interesse, unterschiedliche Modelle der Leistungserbringung und verschiedene Lösungsansätze für die Schnittstellenproblematik zu berücksichtigen. Es werden somit Optionen für einen besseren Zugang für Patienten und eine Reduktion von Abhängigkeiten vom stationären Sektor vorgestellt. Die Länder wurden auch dahingehend ausgewählt, dass sie unterschiedliche sektorenübergreifende Versorgungspfade abbilden. Insbesondere wurden Länder berücksichtigt, die in den letzten Jahren Reformen für eine verbesserte Koordination zwischen den Sektoren und Versorgungsebenen durchgeführt haben, die Potenzial für eine Übertragbarkeit auf Deutschland bieten.

Auf dieser Grundlage wurden Dänemark, Finnland, die Niederlande, Norwegen und Österreich für die weitere Untersuchung ausgewählt und werden in den folgenden thematischen Abschnitten beschrieben.

\subsubsection{Definitionen}

Im Folgenden werden die unterschiedlichen Sektoren und Versorgungsebenen in Gesundheitssystemen beschrieben, an denen sich Schnittstellen befinden und es oft zu Brüchen in den Versorgungspfaden von Patienten kommt.
International wird zwischen den Sektoren der ambulanten und stationären Versorgung unterschieden. Innerhalb dieser Sektoren wird zwischen den Versorgungsebenen der primären, sekundären bzw. fachärztlichen und der tertiären bzw. spezialfachärztlichen Versorgung unterschieden. Zum ambulanten Sektor gehören sowohl die Primärversorgung als auch Teile der Sekundärversorgung. In der stationären Versorgung existiert neben der Sekundärversorgung auch die hochspezialisierte Tertiärversorgung.

\section{- Ambulante Versorgung}

Zur ambulanten Versorgung gehören alle Gesundheitsleistungen, die nicht während eines stationären Aufenthalts erbracht werden. Sie schließen somit Primärversorgung und Teile der Sekundärversorgung mit ein. Ambulante Leistungen sind u. a. ärztliche und zahnärztliche Versorgung, ambulante diagnostische und operative Verfahren, Rehabilitation, Arzneimittelversorgung, Geburtshilfe, erste Hilfe und Notfallversorgung, Gesundheitsprävention und Gesundheitsförderung.

\section{- Primärversorgung}

In den meisten Ländern deckt die Primärversorgung einen Großteil (teils über $90 \%$ ) der Patientenkontakte mit dem Gesundheitssystem ab. Die Primärversorgung wird überwiegend durch Hausärzte sichergestellt. In einigen Ländern werden auch Kinderärzte, Gynäkologen, Zahnärzte oder Psychotherapeuten zu den Primärversorgern gezählt.

Die Primärversorgung ist in Europa sehr unterschiedlich organisiert. In vielen traditionellen Sozialversicherungsländern dominieren Hausärzte, die freiberuflich in Einzelpraxen praktizieren, obwohl Gruppenpraxen, in denen mehrere Ärzte als Angestellte arbeiten (jetzt offiziell „Berufsausübungsgemeinschaften") zunehmend an Bedeutung gewinnen (z. B. Frankreich, Deutschland oder Österreich; s. u.). In Ländern mit einem nationalen Gesundheitsdienst sind Gesundheitszentren oder große Gruppenpraxen weit verbreitet, 


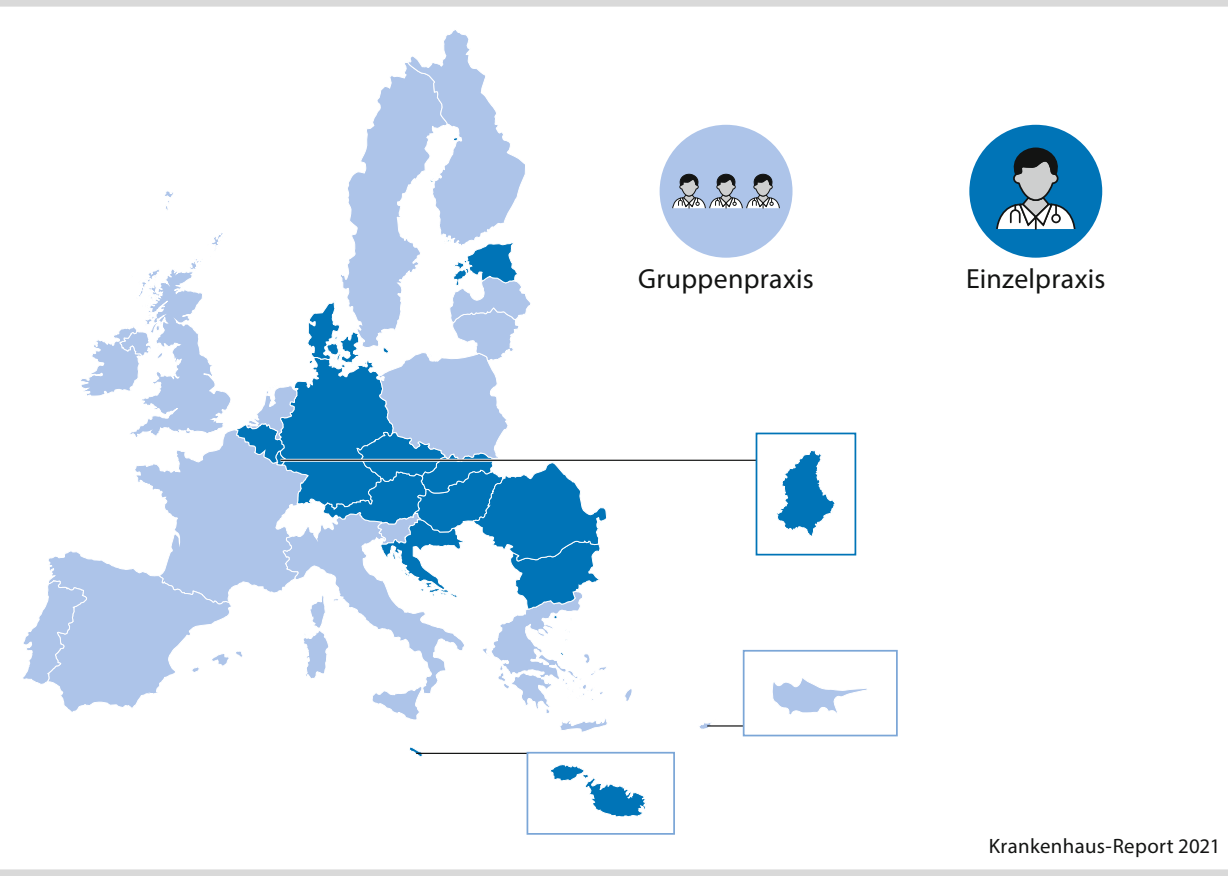

- Abb. 1.1 Vorherrschende Form der ärztlichen Primärversorgung in Ländern der Europäischen Union (Quelle: European Union 2017)

in denen sowohl Hausärzte als auch Krankenpflegekräfte und evtl. weiteres Gesundheitspersonal wie Physiotherapeuten, Ergotherapeuten oder Sozialarbeiter tätig sind (z. B. Finnland, Italien, Norwegen Schweden, Spanien, UK) (s. • Abb. 1.1).

In vielen Ländern agieren Primärversorger als Gatekeeper zu anderen Versorgungsebenen des Gesundheitssystems, d.h. dass Patienten zum Beispiel in Dänemark, Finnland oder den Niederlanden zunächst einen Hausarzt aufsuchen müssen, bevor sie an einen Facharzt überwiesen werden. Im Kontext von alternden Gesellschaften und zunehmend multimorbiden Patienten kommt der Primärversorgung eine immer zentralere Rolle bei der umfassenden, kontinuierlichen und koordinierenden Versorgung zu. In den traditionellen Sozialversicherungsländern ging der Trend in den letzten Jahren dahin, die Gatekeeping-Funktion der Primärversorgung und die Registrierung bei einem Hausarzt zu unterstützen, um die Ko- ordination zwischen Gesundheitsdiensten und die Steuerung der Versorgung zu gewährleisten. In traditionell steuerfinanzierten Ländern liegt der Fokus stärker auf der Einführung alternativer Primärversorgungsformen, wie den sog. ,Walk-in“-Kliniken oder Zentren und der Ausweitung der Wahl des Hausarztes.

\section{- Ambulante Sekundärversorgung}

Ambulante Leistungen werden nicht nur von Primärversorgern erbracht, sondern auch von Spezialisten auf sekundärer Ebene, in der Regel nach Überweisung aus der Primärversorgung. So gehören beispielsweise Fachärzte und anderes medizinisches Fachpersonal in Krankenhausambulanzen, in Einzel- oder Gruppenpraxen oder Dialysezentren zur ambulanten Sekundärversorgung. Neue Technologien und Organisationsmodelle ermöglichen, dass sekundäre Leistungen, die früher einen stationären Aufenthalt erforderlich machten (chirurgische Eingriffe, komplexe diagnosti- 


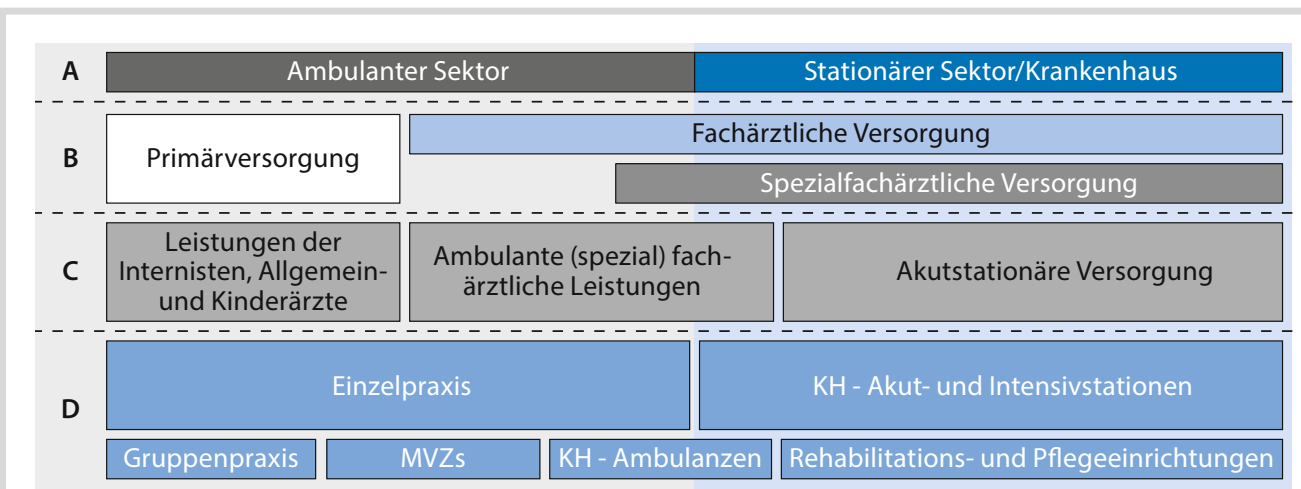

A: Sektor B: Versorgungsebene C: Leistungsspektrum D: Versorgungsformen (Beispiele)

Krankenhaus-Report 2021

- Abb. 1.2 Stark fragmentierte Leistungserbringung in Deutschland: schematische Darstellung nach Sektoren, Versorgungsebenen, Leistungsspektrum und Versorgungsformen. Abkürzungen: KH - Krankenhaus, MVZ - Medizinische Versorgungszentren

sche Verfahren, Chemotherapie oder Rehabilitierung), verstärkt ambulant erbracht werden können. Im Jahr 2011 wurde in Deutschland mit dem GKV-Versorgungsstrukturgesetz der neue Versorgungsbereich der ambulanten spezialfachärztlichen Versorgung geschaffen, in dem Vertragsärzte und Krankenhausambulanzen nach einheitlichen Rechtsvorschriften Patienten mit komplexen, schwer therapierbaren und/oder seltenen Erkrankungen versorgen. Außerdem gibt es in Deutschland noch weitere Rechtsformen, unter denen Krankenhäuser ambulante Leistungen erbringen können (u. a. Psychiatrische Institutsambulanzen, vor- und nachstationäre Behandlung im Krankenhaus, ambulantes Operieren, Pädiatrische Spezialambulanzen) (Leber und Wasem 2016).

\section{n - Stationäre Versorgung}

Die stationäre Versorgung von Patienten erfolgt in Krankenhäusern, Pflegeeinrichtungen oder Reha-Einrichtungen. Stationäre Versorgung setzt voraus, dass Patienten für mindestens eine Nacht in einer dieser Einrichtungen aufgenommen werden. Bei der stationären Versorgung lassen sich zwei Ebenen unterscheiden. Die untere Ebene wird als Teil der Sekundärversorgung gesehen (neben der ambu- lanten Sekundärversorgung), da sie zumindest die zweite Stufe nach der Primärversorgung ist. Zusätzlich gibt es in vielen Ländern die tertiäre Versorgung, womit - zumeist nicht trennscharf zur sekundären Versorgung - hochkomplexe medizinische Leistungen beschrieben werden, die in großen, spezialisierten Krankenhäusern erbracht werden. Tertiäre Leistungen werden meist nach Überweisung durch Fachärzte aus der Sekundärversorgung bzw. Verlegung aus einem Krankenhaus der Sekundärversorgung erbracht. Tertiäre Krankenhäuser versorgen in der Regel eine größere Region mit teureren und aufwendigeren Leistungen.

Auch wenn in Deutschland die gesetzlichen Grundlagen zur Stärkung der sektorenübergreifenden Versorgung und insbesondere die Rechtsformen der ambulanten Behandlung durch Krankenhäuser gestärkt wurden, sind Sektorengrenzen nach wie vor relativ starr (KOMV 2019). - Abb. 1.2 fasst vereinfachend Versorgungsebenen und Versorgungsformen mit ihrem Leistungsspektrum zusammen, um als Grundlage für den Vergleich mit anderen Ländern zu dienen.

So finden in Deutschland effektiv sehr wenige ambulante Leistungen in Krankenhäusern im Vergleich zu anderen europäischen Ländern 
statt. Die Analyse der Ausgaben von einzelnen Leistungserbringergruppen in 19 europäischen Ländern (Geissler et al. 2016) zeigt, dass in Deutschland relativ wenig ambulante Leistungen in Krankenhäusern erbracht werden $(2,8 \%$ der Ausgaben für ambulante Versorgung) im Gegensatz zu Ländern wie Dänemark, Finnland, Portugal, Schweden oder Spanien, wo durchschnittlich 32,4\% auf Ausgaben für ambulante Versorgung in Krankenhäusern entfallen. Die Analyse zeigte insgesamt von 2000 bis 2012 einen deutlichen Anstieg der Ausgaben für ambulante Krankenhausleistungen in den meisten europäischen Ländern.

Der folgende $>$ Abschn. 1.3 beschäftigt sich mit der Etablierung neuer Versorgungsansätze und -formen und Verschiebungen von ambulanter Versorgung in den Krankenhaussektor innerhalb Europas.

\subsection{Verschiebungen von Zuständigkeit, Organisation und Versorgungsprozessen}

In diesem Abschnitt werden anhand von Länderbeispielen (Österreich, Norwegen und Finnland) Reformen bezüglich der Organisation und Neustrukturierung der Gesundheitsversorgung exemplarisch vorgestellt. Außerdem wird der Einsatz ambulanter Operationen als Instrument, das finanzielle, strukturelle und personelle Vorteile bietet, vorgestellt.

\subsubsection{Reformen in europäischen Ländern}

Eine verbesserte Koordination zwischen den Sektoren und Versorgungsebenen wird in vielen europäischen Ländern durch umfassende Reformen - insbesondere auch der Zuständigkeiten - angestrebt. Ziele der Reformen sind eine engere Zusammenarbeit zwischen den Sektoren und Akteuren (Beispiel Österreich, Finnland) und den Versorgungsebenen (primär, sekundär und tertiär) mit dem primären Ziel einer besseren Qualität der Versorgung. Im Folgenden soll aufgezeigt werden, wie Reformen und Entwicklungen in den letzten Jahren in Bezug auf die Verschiebung von Zuständigkeiten und die verstärkte Integration von ambulanter und stationärer Versorgung in Ländern wie Österreich, Norwegen und Finnland stattgefunden haben.

\section{- - Österreich}

Die zentrale Reform des Gesundheitssystems von 2013 in Österreich hatte als wesentliches Ziel die Verbesserung der Koordination und Kooperation zwischen den verschiedenen Akteuren im fragmentierten Gesundheitssystem. Die Reform führte durch eine vertragliche Vereinbarung zwischen dem Bund, den Ländern und den Sozialversicherungsträgern ein zielorientiertes Steuerungssystem ein (,Zielsteuerungsverträge“). Diese Verträge zielten darauf, eine gemeinsame integrative Versorgungsplanung sowie die gemeinsame Steuerung und Finanzierung zu schaffen. Insbesondere sollte auch der Abbau der übermäßigen Abhängigkeit von Krankenhäusern vorangetrieben werden, da Österreich - ähnlich wie Deutschland - ein fragmentiertes Gesundheitssystem mit einem starken Krankenhaussektor hat. Ein weiteres wichtiges Ziel war die Stärkung der Primärversorgung und die Entwicklung eines neuen Primärversorgungskonzepts, das letztlich zur Verabschiedung des Primärversorgungsgesetzes im Jahr 2017 führte. Das Gesetz sieht die Errichtung von multidisziplinären Primärversorgungseinheiten vor, die aus einem Kernteam aus Allgemeinärzten, Krankenpflegern und Praxisassistenten bestehen, die für die Patientenorientierung und die Koordinierung der Leistungen verantwortlich sind. Ziel ist es, eine ganzheitliche und kontinuierliche Patientenbetreuung zu erreichen. Die Primärversorgungseinheiten können auch Kinderärzte und andere Fachkräfte (Physiotherapeuten oder Sozialarbeiter) umfassen. Primärversorgungseinheiten können entweder aus bestehenden Strukturen weiterentwickelt werden oder von Grund auf neu errichtet und 
gestaltet werden. Sie können in Form von Zentren (multiprofessionelles Team in einer Praxis) oder Netzwerken (multiprofessionelle Teamarbeit an unterschiedlichen Standorten) gegründet werden und eng mit anderen Anbietern (Apotheken, Fachärzten, Krankenhäusern, Pflegeheimen, Schulen, Sozialversicherungsträgern usw.) kooperieren (OECD/European Observatory on Health Systems and Policies 2019a).

Auch wenn mit den Zielsteuerungsverträgen ein innovatives Steuerungssystem eingeführt wurde, das es den Hauptakteuren ermöglicht, die zentralen Herausforderungen des österreichischen Gesundheitssystems anzugehen, so blieb jedoch die verfassungsrechtliche Trennung der Zuständigkeiten bestehen. In Österreich sind traditionell die Sozialversicherungsträger für die ambulante Versorgung und die Bundesländer für die Finanzierung und Leistungserbringung der Krankenhäuser verantwortlich (Bachner et al. 2019; Schmidt et al. 2018). Was das Beispiel Österreich vor allem zeigt ist, dass trotz konzertierter und mehrjähriger Zusammenarbeit aller wichtigen Partner im Gesundheitswesen mit dem Ziel, die sektorale Trennung (ambulant vs. stationär) abzuschaffen, es nicht gelungen ist, die Zuständigkeiten neu festzulegen. Jedoch sind die Primärversorgungszentren, die kontinuierlich neu entstehen, ein wichtiger Schritt in Richtung einer stärker koordinierten und integrierten Versorgung. Für Deutschland stellt Österreich aufgrund der Ähnlichkeiten (z.B. starke Selbstverwaltungspartner) ein interessantes Beispiel dar.

\section{- Norwegen}

Das norwegische Gesundheitssystem ist teilweise zentralisiert, der Staat ist verantwortlich für die Sekundärversorgung und die Kommunen für Primärversorgung, Langzeitpflege und soziale Dienste. Eine Reihe von Reformen in den letzten Jahren zielten darauf, die Koordination zwischen den Sektoren und Versorgungsebenen zu verbessern: (1) Die Koordinationsreform von 2012 sollte die Koordination in der Gesundheitsversorgung zwischen Kom- munen und Krankenhäusern verbessern, (2) der nationale Gesundheits- und Krankenhausplan für 2016-2019 zielte auf eine verbesserte Koordination zwischen den Krankenhäusern und (3) der Report (White Paper) „Primärversorgung von morgen“" (2015) erkennt an, dass Lösungen zur Verbesserung der Koordination zwischen Primär- und Sekundärversorgung notwendig sind.

Mit der Koordinationsreform (1) erhielten die Kommunen die Verantwortung, Notfallbetten für Patienten mit Bedarf für vor- und poststationäre Behandlungen/Vor- und Nachbehandlungsleistungen zur Verfügung zu stellen, die allerdings nur für maximal 72 Stunden von einem Patienten, also nicht für Langzeitpflege, genutzt werden dürfen. Mit der Reform erhielten die Kommunen auch mehr Verantwortung für die Koordination der Versorgung, da sie Verträge mit Krankenhäusern für eine angemessene und koordinierte Versorgung von Patienten mit komplexen Bedürfnissen erstellen mussten. Ein zentrales Ziel des nationalen Gesundheits- und Krankenhausplans 2016-2019 (2) war die Verbesserung der Koordination durch Organisation von Krankenhäusern in regionalen Netzwerken. Aufbauend auf den Zielen und Errungenschaften dieser Reformen wird die Stärkung der Primärversorgung für Patienten mit komplexen Bedürfnissen kontinuierlich verfolgt, u.a. durch neue Versorgungsmodelle multidisziplinärer Versorgungsteams (Sperre Saunes et al. 2020b; OECD/ European Observatory on Health Systems and Policies 2019b).

\section{- Finnland}

In Finnland fallen die Organisation und Finanzierung der Gesundheitsleistungen in die Zuständigkeit der Gemeinden. Vom Gesetzgeber sind diese mit der Sicherstellung der Gesundheitsversorgung beauftragt, allerdings existiert keine genaue Definition, wie die Leistungen $\mathrm{zu}$ erbringen sind. Dies liegt allein im Ermessen der Gemeinden, die zunehmend auch bestimmte Leistungen vom Privatsektor einkaufen. Als Verwaltungsbehörden agieren die über 300 Gemeinden damit sowohl als 
„Krankenversicherer“ als auch als Betreiber von Gesundheitszentren und Krankenhäusern. Die Gemeinden schließen sich in 20 Krankenhausbezirken zusammen, die die Träger der öffentlichen Krankenhäuser im jeweiligen Bezirk sind. Die Gemeinden eines Krankenhausbezirks beziehen die notwendigen ambulanten und stationären Leistungen von den Krankenhäusern dieses Bezirks, können aber auch über Ausschreibungen Leistungen von anderen Krankenhausbezirken oder von privaten Kliniken in Anspruch nehmen (Preusker 2019; Keskimäki et al. 2019).

Seit mehr als zehn Jahren wird in Finnland über eine grundlegende Reform des Gesundheit- und Sozialsektors diskutiert, die eine umfassende Gebietsreform mit der Verlagerung der Zuständigkeiten für die Gesundheitsversorgung von Kommunen auf neu einzurichtende Regionen einschließt. Statt der derzeit noch über 300 Kommunen sollen 22 neu geschaffene Regionen die gesundheitliche und soziale Versorgung sicherstellen. Wichtige Aspekte der Reform sind die Förderung einer stärkeren Integration von Diensten und die Einführung von Wahlfreiheit für Patienten bei der Auswahl von Gesundheitsdienstleistern. Das übergeordnete Ziel der Reform ist es, die Erbringung von Dienstleistungen effizienter zu gestalten und die wachsenden Ausgaben für Gesundheitsund Sozialleistungen einzudämmen. Zudem wird die Neuordnung der Krankenhausversorgung mit einer verstärkten Zentralisierung der Akut- und Notfallversorgung, der Konzentration von hochspezialisierten Leistungen sowie der Vorgabe von Mindestmengen angestrebt. Aufgrund der so weitreichenden Änderungen und politischer Verwerfungen über die Inhalte ist das Reformprojekt im März 2019 jedoch kurz vor der Parlamentswahl gescheitert und führte zum Rücktritt der Regierung. Die neue Regierung hat die Reformbestrebungen wieder in ihr Regierungsprogramm aufgenommen (Keskimäki et al. 2019). Der aktuelle Gesetzesentwurf zur Reform der Gesundheits-, Sozial- und Rettungsdienste wird im Dezember 2020 dem Parlament vorgelegt und sieht im Wesentlichen die Umwandlung von Gesund- heitszentren zu integrierten Gesundheits- und Sozialzentren vor (Sote-uudistus 2020).

\subsubsection{Tagesfälle und ambulante Operationen in Europa}

Fast alle Länder experimentieren mit der ReOrganisation der Gesundheitsversorgung und dem Verschieben von Zuständigkeiten zwischen den Sektoren und den verschiedenen Ebenen, um die Kontinuität und Qualität der Versorgung und von Informationen zu verbessern, die Wahlmöglichkeiten der Patienten $\mathrm{zu}$ erhöhen und die kontinuierlich steigenden Kosten abzudämpfen. Um Übergänge zwischen den Sektoren zu verbessern und dem Koordinationsbedarf von Patienten gerecht zu werden, haben die meisten europäischen Länder in den letzten Jahrzehnten verstärkt den Krankenhaussektor reformiert und versucht, neue Versorgungsansätze und -formen zu etablieren, in denen Patienten ambulant im Krankenhaus behandelt werden können (ambulante Operationen) oder die Versorgung durch ambulante Gesundheitsdienstleister erbracht wird. In vielen Ländern ersetzen ambulante Leistungen dadurch verstärkt die stationäre Versorgung (Nair et al. 2020; OECD 2018). Die Anteile von ambulant durchgeführten operativen Eingriffen sind im letzten Jahrzehnt in den meisten europäischen Ländern gestiegen, jedoch unterschiedlich stark. Daher variieren die Anteile der Tagesfälle zwischen den Ländern (OECD 2018) und korrelieren oft mit den Anteilen an ambulanten Leistungen im Krankenhaus. Die nordischen Länder, aber auch die Niederlande und das Vereinigte Königreich haben bei der Einführung der Tagesoperation eine Vorreiterrolle gespielt; eine wachsende Anzahl von Operationen werden dort ambulant durchgeführt (OECD 2018; Geissler et al. 2016). In Finnland wird beispielsweise seit den 1990er Jahren eine Verlegung von ambulanten Operationen in den tagesklinischen Bereich angestrebt. Dazu sind in vielen Krankenhäusern spezifische ambulante Operations- 
zentren sowie Tageskliniken entstanden. Das Land weist den stärksten Anstieg von ambulant durchgeführten Tonsillektomien zwischen 2000 und 2016 im Vergleich zu 23 anderen EU-Ländern auf sowie die größten Anteile an ambulanten Operationen für Katarakt- und Mandeloperationen mit $98,8 \%$ bzw. $86,6 \%$ gegenüber dem Durchschnitt von $84,2 \%$ bzw. 29,2\% von 24 EU-Ländern (OECD 2018). Heutzutage machen ambulante Behandlungsverfahren dort etwas weniger als die Hälfte aller chirurgischen Eingriffe aus, wenn auch mit großen Unterschieden zwischen den Krankenhausbezirken (Preusker 2019). Mehrere Länder in West- und Südeuropa waren mäßig schnelle Anwender von Tagesoperationen. In Österreich, Deutschland und mehreren Ländern Mittel- und Osteuropas (z. B. Ungarn, Polen und Rumänien) war die Verbreitung der Tagesoperation für die meisten Eingriffe allgemein viel langsamer (OECD 2018).

\subsection{Neue Formen und Prozesse der Zusammenarbeit im ambulanten und stationären Sektor: Leistungserbringung und Patientenpfade in europäischen Ländern}

Auch wenn viele Länder ähnliche Ziele hinsichtlich einer verbesserten Koordination und Kommunikation an den Schnittstellen zwischen den Sektoren und Versorgungsebenen verfolgen, sind die strukturellen Ausgangspositionen sehr verschieden. Jedes Land hat aufgrund der historischen Entstehung und kontextueller Faktoren eine andere Organisation der Zuständigkeiten und andere Strukturen und Funktionen der Leistungserbringung im Gesundheitssystem. Damit unterscheidet sich auch, wie sich Patientinnen und Patienten in dem jeweiligen Gesundheitssystem bewegen und mit Gesundheitsanbietern interagieren, d. h. wo und von wem sie welche Leistung er- halten. Anhand von Länderbeispielen aus den Niederlanden, Norwegen und Finnland soll in diesem Abschnitt aufgezeigt werden, wie Prozesse in den Bereichen der intersektoralen Organisation, Kommunikation und Arbeitsabläufe an den Schnittstellen zwischen ambulanten und stationären Leistungserbringern gestaltet sind. Mit Hilfe von detailliert dargestellten Patientenpfaden aus Finnland und den Niederlanden wird exemplarisch dargestellt, wie unterschiedlich Versorgungsketten in verschiedenen europäischen Ländern aufgebaut sind.

\section{- Niederlande}

Die Primärversorgung in den Niederlanden umfasst eine Vielzahl von Leistungserbringern, darunter Hausärzte, Physiotherapeuten, Apotheker, Psychologen und Hebammen. Hausärzte spielen eine zentrale Rolle in der Primärversorgung und im Gesundheitssystem im Allgemeinen, da sie als „Gatekeeper“ fungieren. In Kasten 1 wird der Patientenpfad innerhalb der Primärversorgung exemplarisch für einen multimorbiden Patienten detailliert beschrieben. Alle Bürgerinnen und Bürger sind bei einem Hausarzt eingeschrieben, der in Wohnortnähe tätig ist. Eingeschriebene Patienten haben so die Möglichkeit, außerhalb der regulären Öffnungszeiten des Hausarztes das Notfallangebot in Anspruch zu nehmen. Fast alle Hausärzte $(82 \%)$ arbeiteten 2016 in kleinen Praxen von zwei bis sieben Ärzten; nur $18 \%$ arbeiteten in Einzelpraxen (Wammes et al. 2020; Kroneman et al. 2016).

Die Sekundärversorgung umfasst jene Versorgungsformen, die nur auf Überweisung eines Primärversorgers zugänglich sind. Diese Formen der Versorgung werden hauptsächlich von Krankenhäusern und psychiatrischen Einrichtungen erbracht, denn Fachärzte sind zu einem Großteil in Krankenhäusern tätig. Krankenhäuser verfügen sowohl über stationäre und ambulante Abteilungen als auch über 24-Stunden-Notfallstationen. Ambulante Abteilungen werden auch für die Diagnose vor oder nach einem Krankenhausaufenthalt genutzt. Innerhalb der Krankenhäuser sind etwa $60 \%$ der Fachärzte selbstständig. In ei- 


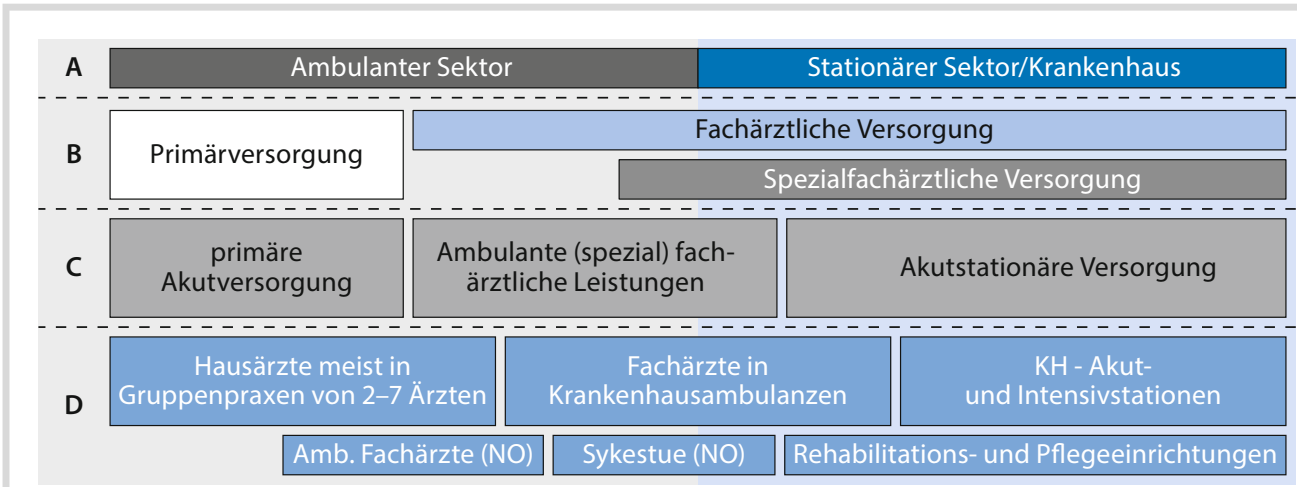

A: Sektor

B: Versorgungsebene

C: Leistungsspektrum

D: Versorgungsformen (Beispiele)

Krankenhaus-Report 2021

- Abb. 1.3 Ambulante fachärztliche Versorgung im Krankenhaus: schematische Darstellung nach Sektoren, Versorgungsebenen, Leistungsspektrum und Versorgungsformen in den Niederlanden und Norwegen

nigen wenigen Krankenhäusern, insbesondere in Universitätskliniken, sind alle Fachärzte im Krankenhaus angestellt. Darüber hinaus sind alle Kinderärzte in Krankenhäusern im Angestelltenverhältnis tätig (Kroneman et al. 2016). - Abb. 1.3 beinhaltet eine schematische Darstellung der ambulant-fachärztlichen Versorgung im Krankhaus in den Niederlanden.

Bei der Organisation der Notfallversorgung findet eine verstärkte Koordination zwischen ambulanter und stationärer Versorgung statt. Denn hier nehmen ambulant tätige Hausärzte die entscheidende Triage-Funktion in Krankenhäusern ein und steuern damit den Fluss der Patienten, die entweder stationär oder ambulant behandelt werden (Nagel et al. 2017; Kroneman et al. 2016; OECD/European Observatory on Health Systems and Policies 2019c).

\section{Kasten 1 - Patientenpfad eines multimorbiden Patienten in den Niederlanden}

Die Verantwortung für die Koordination der Versorgung eines multimorbiden Patienten liegt in den Niederlanden beim Hausarzt. Wenn jedoch der größte Teil der Behand- lungen durch einen Facharzt erfolgt, wird die Koordination von diesem übernommen. Je nach Komplexität der Erkrankungen sind andere Leistungserbringer der Primär- und/ oder Sekundärversorgung an der Versorgung beteiligt (z. B. Gemeindepflegekraft, Physiotherapeut, Ergotherapeut, Facharzt). In der Regel ist eine Krankenpflegekraft aus der Primärversorgung ebenfalls an der Versorgung beteiligt.

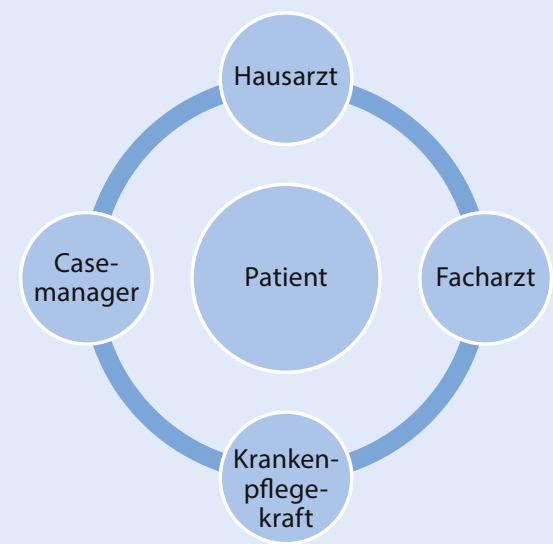

Der Hausarzt übernimmt regelmäßige Untersuchungen (Überprüfung der Medikati- 
on gemeinsam mit dem Apotheker) zur Überwachung des Gesundheitszustandes, zur Behandlung und zur gemeinsamen Vereinbarung individueller Behandlungspläne, die zunehmend Verwendung finden. Diese Behandlungspläne, die Prioritäten bei der Behandlung, Zielvorgaben und Selbstmanagementaktivitäten beinhalten, werden regelmäßig mit dem Patienten besprochen und aktualisiert. Damit werden sich die Behandlungs- und Versorgungsziele im Verlauf der Erkrankung und je nach Auftreten von Komplikationen, Prognose und Lebenserwartung immer wieder verändern.

Leidet der Patient unter einer der folgenden chronischen Erkrankungen, wird eine nationale Versorgungsleitlinie angewendet: Diabetes mellitus, COPD oder HerzKreislauf-Erkrankung. Ein Case Manager wird hinzugezogen, wenn die Koordination zu komplex wird, da beispielsweise $\mathrm{zu}$ viele Leistungserbringer beteiligt sind, Widersprüche in den Behandlungsplänen entstehen oder die Resilienz des Patienten nicht ausreichend ist. Der Case-Manager ist in der Regel eine Krankenpflegekraft der primären Gesundheitsversorgung, deren Aufgabe es primär ist, den Patienten sowie pflegende Angehörige beim Versorgungsmanagement zu unterstützen und anzuleiten. (Quelle: Kroneman et al. 2016)

\section{- Norwegen}

In Norwegen sind die Kommunen für die Primärversorgung verantwortlich. Sie können frei entscheiden, wie die Versorgung organisiert werden soll; dazu gehört auch, ob sie Hausärzte als öffentliche Angestellte einstellen oder Verträge mit privaten Ärzten abschließen. Die meisten Hausärzte sind selbstständig, arbeiten in hausärztlichen Gemeinschaftspraxen und im Auftrag der Kommunen. Eine typische Praxis besteht normalerweise aus zwei bis sechs Ärzten und Hilfskräften. Jeder Norweger ist angehalten, sich fest bei einem Hausarzt einzu- schreiben, der auch als „Gatekeeper“ fungiert. Sollte der Hausarzt im akuten Krankheitsfall keinen freien Termin haben oder außerhalb der Sprechzeiten nicht zugänglich sein, stehen in allen großen und mittelgroßen Städten und Orten rund um die Uhr Ambulanzzentren (sog. Legevakt) zur Verfügung.

Eine ambulante fachärztliche Versorgung wird normalerweise in ambulanten Krankenhausabteilungen angeboten, die als Polikliniken bezeichnet werden, sowie durch selbständige, privat praktizierende Fachärzte (z. B. Geburtshelfer, Fachärzte für Innere Medizin), die im Rahmen einer vertraglichen Vereinbarung mit den nationalen Gesundheitsbehörden meist in ihren eigenen Praxen arbeiten. Auf letztere entfallen etwa $25 \%$ aller ambulanten Fachkonsultationen. In den ländlichen und entlegeneren Teilen des Landes erfolgt die Versorgung in kommunalen Krankenhäusern. Sie bieten Leistungen an, die nicht am Wohnort des Patienten in Anspruch genommen werden können, aber keine Krankenhauseinweisung sowie eine Nachsorge nach dem Krankenhausaufenthalt erfordern, und entscheiden, ob ein Krankenhausaufenthalt in einem Akutkrankenhaus erforderlich ist. Diese Einrichtungen sind häufig zusammen mit anderen kommunalen Gesundheitsdiensten untergebracht. Eine Reihe von Behandlungen werden in Norwegen heute nur noch vorwiegend in Form ambulanter Tagespflege angeboten, darunter somatische Behandlungen (z. B. Operationen), psychiatrische Betreuung (z.B. Behandlung von Essstörungen) und Behandlung von Drogen- und Alkoholabhängigkeit. - Abb. 1.3 beinhaltet eine schematische Darstellung der ambulantfachärztlichen Versorgung im Krankenhaus in Norwegen.

Sekundäre Rehabilitationsleistungen werden in Krankenhäusern, in speziellen Rehabilitationsabteilungen oder in anderen Einheiten wie Rheumatologie- oder Neurologieabteilungen erbracht (Sperre Saunes et al. 2020a).

\section{- Finnland}

Patienten in Finnland, die keinen medizinischen Notfall darstellen, wenden sich in der 


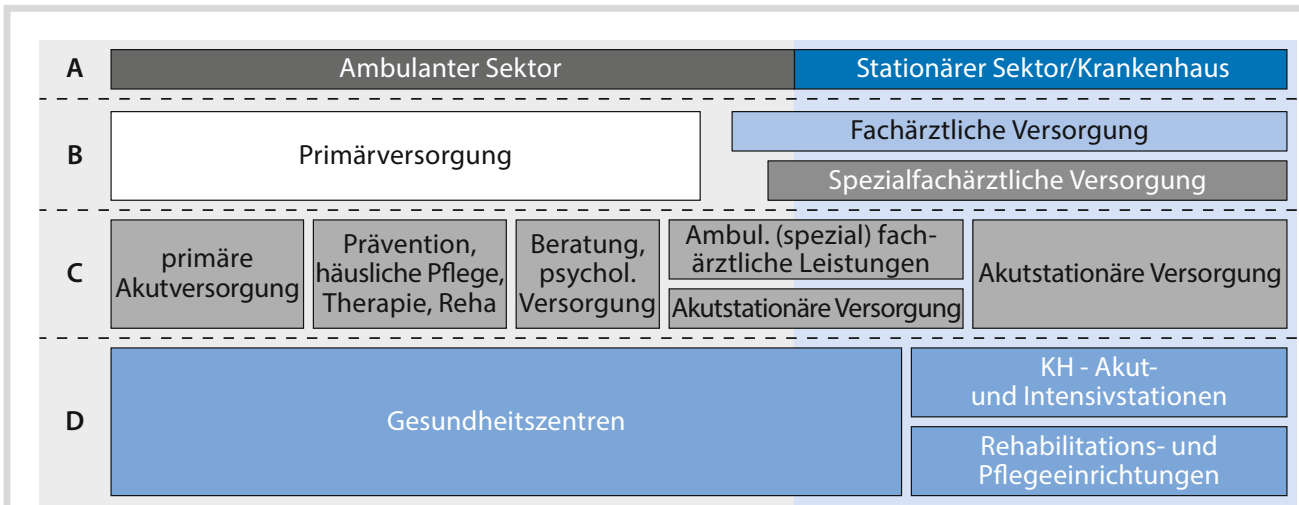

A: Sektor B: Versorgungsebene C: Leistungsspektrum D: Versorgungsformen (Beispiele)

Krankenhaus-Report 2021

- Abb. 1.4 Stationäre Abteilungen in Gesundheitszentren in Finnland: schematische Darstellung nach Sektoren, Versorgungsebenen, Leistungsspektrum und Versorgungsformen

Regel an die Gesundheitszentren ihrer Gemeinde, die die Grundversorgung der Bevölkerung mit präventiven und kurativen Leistungen und Vorsorgeleistungen sicherstellt. Gesundheitszentren bezeichnen vorwiegend Hausarztpraxen, in denen auch Internisten, Kinderärzte, Krankenschwestern und Krankenpflegespezialisten (sog. Public Health Nurses und zum Teil auf chronisch Kranke spezialisiertes Pflegepersonal) beschäftigt sind. Niedergelassene Ärzte in eigenen Praxen existieren in Finnland dagegen nicht. Die landesweit etwa 150 Gesundheitszentren sind entweder im Besitz einer oder mehrerer Gemeinden (Ministry of Social Affairs and Health 2020). Die Leistungserbringung von Gesundheitszentren kann an verschiedenen Orten stattfinden: entweder in Gesundheitszentren selbst, in einer Klinik oder zunehmend auch dezentral, also am Wohnort des Patienten. Die Leistungen eines Gesundheitszentrums erstrecken sich auf ambulante und stationäre Versorgung: (1) ambulante Versorgung sowohl für akut als auch chronisch Kranke; (2) Präventionsdienste einschließlich Schwangerschaftsvorsorge und Kinderkliniken; (3) häusliche Pflege für ältere Patienten oder für ausgewählte Gruppen chronisch Kranker; (4) zahnmedizinische Versorgung; (5) Rehabilitation in verschiedenen For- men; (6) psychiatrische Dienste und Drogenmissbrauchsdienste. Gesundheitszentren verfügen in der Regel über einen Bestand an Medikamenten für den Eigenbedarf. Je nach Gemeinde können in Gesundheitszentren andere Dienstleistungen wie Physiotherapie, Psychotherapie, Logo- und Sprachtherapie, Ergotherapie und medizinische Fachberatung angeboten werden. Größere Gesundheitszentren sind in der Regel gut mit Personal und medizinischer Technologie ausgestattet. Sie haben routinemäßigen Zugang zu anderen Fachärzten, zum Beispiel zur Interpretation radiologischer Untersuchungen. Ein Austausch erfolgt hier häufig über sog. Remote-Dienste, da die Digitalisierung in Finnland eine zentrale Rolle spielt. Zusätzlich verfügen sie über radiologische Einrichtungen, Laboratorien für Probenentnahmen, andere Diagnosegeräte (z. B. für die Durchführung von Elektrokardiogrammund Ultraschalluntersuchungen) und Einrichtungen für kleinere chirurgische und endoskopische Untersuchungen (Keskimäki et al. 2019).

Stationäre Abteilungen in Gesundheitszentren sind ein besonderes Merkmal der finnischen Grundversorgung (s. • Abb. 1.4). Im Jahr 2015 gab es 226 dieser stationären Krankenstationen, die mit Krankenschwestern be- 
setzt waren und von einem Hausarzt, Beleghausarzt oder Facharzt für Geriatrie geleitet wurden. Auf diese Einheiten entfallen etwa 20-25\% aller akutstationären Aufnahmen. Eine typische stationäre Einrichtung eines Gesundheitszentrums verfügt über 30 bis 60 Betten. In größeren Städten wie Helsinki sind sie jedoch noch größer und schließen medizinische Fachkräfte in das feste Mitarbeiterteam ein. Diese Stationen werden seit langem zur Langzeitbehandlung älterer Patienten mit chronischen Krankheiten eingesetzt. Während des letzten Jahrzehnts haben diese von Hausärzten geführten Einrichtungen aufgrund der Zentralisierung der Facharztversorgung und der veränderten Betreuung älterer Patienten eine aktivere Rolle übernommen, z. B. im Bereich der Rehabilitation und in einigen Teilen der Facharztversorgung (z. B. Krebsbehandlung). Gegenwärtig werden diese Stationen häufig zu gleichen Teilen für die Akut- und die chronische Versorgung genutzt, wobei einige Betten für Patienten reserviert sind, die an Demenz leiden oder anderweitig zeitweise betreut werden müssen. Alle Gesundheitszentren verfügen ebenfalls über eine Notfallambulanz während der Öffnungszeiten, deren Besetzung durch Hausärzte oder Krankenschwestern sichergestellt wird. Seit 2013 wird die Notfallambulanz außerhalb der Sprechstunden durch Kliniken sichergestellt. Die spezialisierte ambulante Versorgung erfolgt hauptsächlich in ambulanten Abteilungen öffentlicher Krankenhäuser oder bei kleineren Behandlungen und - wenn das erforderliche Fachwissen verfügbar ist - in größeren Gesundheitszentren. Die stationäre Versorgung wird größtenteils von Krankenhausbezirken erbracht. Die Rehabilitation im Gesundheitswesen erfolgt je nach Pflegebedarf des Patienten in Krankenhäusern, Gesundheitszentren und Einrichtungen für betreutes Wohnen oder ambulant (Keskimäki et al. 2019; Preusker 2019). Durch die Primärversorgungszentren mit multiprofessioneller Ausrichtung und ihrem umfassenden Leistungsprofil (Gesundheitsförderung, Prävention, Diagnose, Therapie, soziale, rehabilitative, edukative Leistungen) wird eine integrier- te Versorgung ermöglicht. Die Beschreibung des Patientenpfades einer Patientin mit elektiver Hüftprothese verdeutlicht ein Bespiel einer Versorgungskette in Finnland (Kasten 2).

Kasten 2 - Patientenpfad einer

Patientin mit elektiver Hüftprothese in Finnland

1. Termin Gesundheitszentrum Besteht Bedarf zur Terminvereinbarung, ruft die Patientin direkt ihr zuständiges Team (bestehend aus Krankenschwester und Hausarzt) im Gesundheitszentrum ihrer Wohngemeinde an. Innerhalb von einigen Wochen erhält sie daraufhin einen Termin bei ihrem Hausarzt im Gesundheitszentrum (eine Online-Terminvergabe ist zunehmend möglich). Während des Termins beurteilt der Hausarzt den Gesundheitszustand der Patientin, ordnet ein Röntgenbild der Hüfte, Laboruntersuchungen und ggf. Medikamente und Physiotherapie an.

2. Termin Gesundheitszentrum Im Gesundheitszentrum wird eine mögliche Verschlechterung oder Verbesserung des Gesundheitszustandes geprüft und in einem gemeinsamen Entscheidungsfindungsprozess mit der Patientin ein möglicher operativer Eingriff besprochen. Daraufhin schreibt der Hausarzt eine Überweisung zum Orthopäden, über den einige Gesundheitszentren verfügen. Muss die Patientin ein öffentliches Krankenhaus mit orthopädischen Leistungen aufsuchen, das von dem Krankenhausbezirk betrieben wird, zu dem ihre Wohngemeinde gehört, ist es möglich, dass die Patientin drei Monate oder länger auf ihren Facharzttermin warten muss.

\section{Termin beim Facharzt, Operation und} Nachsorge Letztlich beurteilt der orthopädische Chirurg, ob eine Operation notwendig ist, nachdem nochmals überprüft wird, ob nicht-chirurgische Maßnahmen wie z. B. 
Gewichtsabnahme die Symptome nicht bereits gebessert haben. Nach erfolgter Operation und ersten Rehabilitationsmaßnahmen (Physiotherapie) im Krankenhaus wird die Patientin entlassen. Abhängig von den $\mathrm{Zu}-$ ständigkeiten innerhalb der Gemeinde der Patientin überprüft z. B. ein Physiotherapeut ihre Wohnung auf notwendige Anpassungen wie das Entfernen von Stolperfallen und den Einsatz von Hilfsmitteln. Dieser setzt die Therapie nach der Entlassung fort. Andere vom Krankenhaus verordnete ambulante Pflegeleistungen werden von der Gemeinde gegen eine geringe, einkommensabhängige Gebühr erbracht. Der Hausarzt erhält eine Entlassungszusammenfassung vom Krankenhaus und die Erstversorgungsschwester entfernt auf Anweisung des Chirurgen die Fäden und kontrolliert die Wunde. Wahrscheinlich wird ein anschlieBender Krankenhausbesuch stattfinden, um das Ergebnis des Verfahrens zu überprüfen. (Quelle: Keskimäki et al. 2019)

\subsection{Zentrale Bereiche zur Überwindung von Schnittstellenproblemen}

Das Interesse an einer Weiterentwicklung von Versorgungsprozessen, die darauf zielen, die ambulante und stationäre Versorgung neu zu strukturieren und stärker zu vernetzen, spiegelt sich in den untersuchten Ländern in den Reformen wider. Diese reichen von gesetzgeberischen Maßnahmen, um die stationäre Versorgung verstärkt durch ambulante Leistungen $\mathrm{zu}$ ersetzen, bis hin zu selektiven Kooperationsverträgen, um eine verbesserten Koordination und Kommunikation an den Schnittstellen zwischen den Sektoren und Versorgungsebenen zu verfolgen. Die Bereiche Steuerung und Zuständigkeiten, Finanzierung und Digitalisierung sind bei Sicherstellung der Versorgungskontinuität an Schnittstellen zentral. Im folgenden Abschn. 1.5.1 wird dargestellt, wie diese drei Kernbereiche bereits als Lösungsoption in anderen europäischen Ländern eingesetzt werden und dadurch wichtige Voraussetzungen geschaffen worden sind, um fortbestehende Schnittstellenprobleme zu beheben oder zumindest erheblich zu verringern.

\subsubsection{Lösungsansätze für den Bereich Steuerung und Zuständigkeiten aus Finnland, den Niederlanden und Norwegen}

Die Verteilung der Zuständigkeiten für die ambulante und stationäre Versorgung, die für die Primär-, Sekundär- und Tertiärversorgung verantwortlich sind, ist zentral für die Bewältigung bestehender Schnittstellenprobleme zwischen der ambulanten und der stationären Gesundheitsversorgung. In Deutschland sind für die Sicherstellung der ambulanten Versorgung primär die Kassenärztlichen Vereinigungen zuständig, für die stationäre Versorgung, die Krankenhausplanung und -investitionen liegt die Verantwortung hingegen bei den Bundesländern. Für die Bereiche der ambulanten Sekundärversorgung sowie für Prävention und Gesundheitsförderung sind mehrere Akteure zuständig. Die damit einhergehende Interessenheterogenität zwischen den Interessenvertretern der Leistungserbringer erschwert eine effektive Handlungskoordinierung zwischen den Sektoren. Spezielle Versorgungsformen wie die ambulante spezialfachärztliche Versorgung und Kooperationsverträge von Krankenhäusern mit niedergelassenen Ärzten sind Ansätze, die bereits existieren, eine sektorenübergreifende Versorgung begünstigen und erfolgreich punktuell/regional umgesetzt werden (SVR 2018). Jedoch sind diese Modellvorhaben und Initiativen meist unzusammenhängende Einzelmaßnahmen (KOMV 2019). Derzeit fehlt es in Deutschland an einem ordnungspolitischen Konzept, mit dem umfassen- 
de sektorenübergreifende Strategien umgesetzt werden, die von der Bedarfsplanung, Vergütung, Mengensteuerung, Zulassung und Versorgungssteuerung bis hin $\mathrm{zu}$ einzelvertraglichen Möglichkeiten reichen.

Die aufgeführten Beispiele aus europäischen Ländern wie Finnland, die Niederlande und Norwegen zeigen, welche grundlegenden Umstrukturierungen in der Versorgungslandschaft und Ausweitungen der Versorgungsaufträge der Krankenhäuser und niedergelassenen Ärzte notwendig sind, um eine teilweise Überwindung sektoraler Grenzen zu erreichen. Die Versorgungsbeispiele zeigen auch, wie Krankenhäuser regional stärker vernetzt sein und kooperieren können. Ferner haben die Reformen gezeigt, dass Verschiebungen von Zuständigkeiten notwendig sind, um die sektorale Trennung im Schnittstellenbereich von ambulant und stationär zu überwinden.

\subsubsection{Lösungsansatz für den Bereich Finanzierung aus den Niederlanden}

Eine sektorenübergreifende Finanzierung bedarf adäquater Finanzierungsquellen und angemessener Vergütungsmethoden (Anderson 2011) sowie gleicher Rahmenbedingungen (Leistungsdefinitionen, Qualitätssicherung, einheitliche Art der Kodierung und Leistungsdokumentation) zwischen dem ambulanten und dem stationären Sektor. Derzeitig gängige Vergütungsmechanismen im deutschen Gesundheitswesen reichen für eine qualitativ hochwertige und koordinierte Versorgung an den Schnittstellen nicht aus und bieten nicht genügend Anreize für eine sektorenübergreifende oder integrierte Versorgung. Insbesondere an der ambulant-stationären Schnittstelle treffen mit dem EBM, der GOÄ und dem DRG-System drei verschiedene Vergütungssystematiken aufeinander, zusätzlich $\mathrm{zu}$ den Vergütungsregelungen für besondere Versorgungsformen (SVR 2018; KOMV 2019). Daher ist es wichtig, ein sektorenüber- greifendes Vergütungssystem zu entwickeln und $\mathrm{zu}$ implementieren, das eine bessere Koordination sowie Integration der Versorgung fördert (Struijs et al. 2011; SVR 2018).

Lösungsansätze für sektorenübergreifende Vergütung sind u. a. sog. bundled payments, die in den Niederlanden seit 2015 Anwendung finden. Dort wurde ein neues Vergütungssystem eingeführt, das aus drei Segmenten besteht: (1) Das erste Segment setzt sich aus einer Kopfpauschale und Einzelleistungsvergütung zusammen und (2) das zweite Segment stellt die Grundlage der Vergütung von (mehrfach) chronischen Erkrankungen im Rahmen einer integrierten und multidisziplinären Versorgung dar. Für eine schnittstellenfreie Versorgung schließen sich hier Haus- und Fachärztinnen und -ärzte zu sog. „Care Groups“ zusammen (zwischen 4 und 150 Ärzten). Die Leitung und Koordination der Care Groups übernehmen die Hausärzte. Die Vergütung wird in diesem Fall durch eine diagnosebezogene Pauschale (bundled payments) bestimmt. Diese wird zwischen der „Care Group“ und den Krankenkassen ausgehandelt und aufwandsorientiert - nach Anteil am gesamten Behandlungsprozess - unter den Mitgliedern der Care Group verteilt (IGES 2019). Durch die Einführung der interdisziplinären Behandlung und Vergütung von Care Groups im Rahmen des zweiten Segments sind die Krankenhauseinweisungen chronisch erkrankter Patientinnen und Patienten deutlich zurückgegangen (Czypionka et al. 2015). Bei ambulanten Behandlungen im Krankenhaus wird die Vergütung entweder durch eine diagnosebezogene Pauschale im Rahmen einer Care Group bestimmt oder zwischen Krankenhaus und Fachärztin/-arzt verhandelt (de Graaff und Spee 2018). (3) Das dritte Segment umfasst Selektivverträge zwischen Krankenkassen und Leistungserbringern und zielt dabei insbesondere auf die Förderung von Innovationen und der Qualität der Versorgung ab und ist daher Outcome-bezogen.

Die Ergebnisse des EU-Projekts ICARE4EU, das unter anderem die Finanzierungsmechanismen integrierter Versorgungsprogramme für multimorbide Menschen erhoben hat, 
haben gezeigt, dass innovative Vergütungsansätze, die potenziell zur Förderung von sektorenübergreifender Versorgung genutzt werden könnten, (1) koordinations- bzw. kooperationsorientierte Vergütung, die beispielsweise die Häufigkeit der Überweisung an andere Leistungserbringer positiv beeinflusst, (2) Gewinnausschüttungen, bei denen der erzielte Gesundheitsnutzen stärker im Fokus steht, und (3) Komplexpauschalen in Kombination mit verschiedenen Vergütungsansätzen, die in einem bestimmten Land funktionieren (z. B. Budgets, Kopfpauschale, Fallpauschalen und Einzelleistungsvergütung) umfassen (Struckmann et al. 2017).

Ebenso ist eine Weiterentwicklung der Krankenhausvergütung wichtig, wie das Gutachten des Sachverständigenrates 2018 hervorgehoben hat (SVR 2018), insbesondere die Integration von Vorhaltepauschalen für Leistungen in bedarfsnotwendigen Krankenhäusern, die unabhängig von der Fallzahl erbracht werden müssen. Darauf aufbauend könnten durch Pauschalen für sektorengleiche Leistungen, beispielsweise zur Erbringung von Operationen und anderer stationärer Leistungen, Anreize geschaffen werden, diese künftig ambulant zu erbringen. Derartige Veränderungen benötigen jedoch klare finanzielle Anreize und entsprechende Vorgaben durch den Gesetzgeber, die eingehalten werden müssen.

\subsubsection{Lösungsansätze für den Bereich Digitalisierung aus Estland, Dänemark und den Niederlanden}

Durch den Einsatz digitaler Lösungen, z. B. in Form einer elektronischen Patientenakte, ist es möglich, eine sektorenübergreifende Koordination und Kommunikation zu optimieren und bestehende Barrieren $\mathrm{zu}$ überwinden, bei gleichzeitiger Steigerung der Effizienz und Versorgungsqualität im Gesundheitssystem (Schneider 2016). Wird der Prozess der Überwindung der sektoralen Versorgungshin- dernisse mit der weiteren Einführung der Digitalisierung kombiniert, sind die Effekte zur Steigerung von Effizienz und Effektivität umso stärker. Strukturähnliche Länder wie beispielsweise Dänemark oder Estland sind bei der Einführung elektronischer Patientenakten bereits viel weiter als Deutschland. Estland setzte bei der Umsetzung seiner E-Health-Strategie auf einen Mix aus gesetzlichen Vorschriften sowie finanziellen Anreizen und Sanktionen, um Leistungserbringer zur Mitarbeit zu motivieren (Lai et al. 2013). Außerdem wurde die Einführung der elektronischen Patientenakte durch eine bereits vorhandene digitale Infrastruktur in der öffentlichen Verwaltung stark gefördert. Inzwischen sind alle rund 50 estnischen Krankenhäuser Bestandteil des E-Health-Systems, ebenso fast alle Hausund über die Hälfte der Facharztpraxen. Sie sind in der Lage, die elektronische Patientenakte einzusehen, um aktuelle Diagnose- und Behandlungsinformationen zu ergänzen oder Medikationspläne von Patienten zu aktualisieren. Das umfangreiche E-Health-System spart Zeit und Geld, z. B. indem unnötige Doppeluntersuchungen vermieden werden. Die Esten haben eine ,Opt-out"-Option, das heißt sie können sich der Teilnahme verweigern; die Nutzung der elektronischen Patientenakte und der elektronischen Abrechnung ist für Leistungserbringer allerdings verpflichtend (Bertram et al. 2019).

In Dänemark wurde mit der Einführung einer elektronischen Patientenakte und von ERezepten bereits im Jahr 2003 parallel auch eine Integration aller Versorgungsbereiche vorangetrieben. Das staatlich finanzierte internetbasierte Gesundheitsportal sundhed.dk stellt hier die Schnittstelle aller Digital-HealthAnwendungen dar, führt die Informationen verschiedener Leistungserbringer zusammen und dient als gemeinsame Kommunikationsplattform. Die Plattform wird u.a. zu Abrechnungszwecken verwendet, von Patientinnen und Patienten, um Zugang zu ihren eigenen Gesundheitsdaten zu erhalten, oder von Apotheken, wenn sie Rezepte erhalten. Patient haben durch die Plattform Zugang zu 
ihrer eigenen elektronischen Gesundheitsakte (Sundhedsjournalen), die aktuellste Informationen über bisherige Behandlungen und Diagnosen enthält, und zu ihrer eigenen elektronischen Krankenhausakte (e-jornal), die Informationen zu Behandlungen in den öffentlichen Krankenhäusern sammelt. Außerdem haben sie die Möglichkeit, Termine bei ihrem Hausarzt zu buchen, verschreibungspflichtige Medikamente $\mathrm{zu}$ erneuern, die eigene Medikamenten-Compliance zu überwachen und Wartelisten für Operationen sowie Qualitätsbewertungen von Krankenhäusern einzusehen (Sundhed.dk 2020).

In den Niederlanden hatte die Einführung der digitalen Plattform „ZorgDomein“ vor mehr als 15 Jahren das Ziel, durch eine stärkere Integration von Hausärzten, Krankenhäusern und anderen sekundären Gesundheitsdiensten die Versorgung besser zu koordinieren. Es findet ein digitaler und standardisierter Informationsaustausch zwischen Krankenhäusern und Hausärzten und Krankenhäusern und Leistungsanbietern häuslicher Pflege statt. Dieser wird von ZorgDomein koordiniert und Hausärzte überweisen ihre Patienten über das sog. eReferral-System, das den Hausarzt während des gesamten Prozesses unterstützt. Das eReferral-System verfügt über eine Schnittstelle zur elektronischen Patientenakte des Hausarztes, um eine doppelte Registrierung zu vermeiden und die Datenbank der elektronischen Patientenakte nach der Einweisung, einer Anfrage oder dem Arztbesuch auf dem neuesten Stand zu halten. Somit wird der Informationsaustausch und die Kommunikation von Patienten und allen an der Versorgung beteiligten Leistungserbringern verbessert. $\mathrm{Pa}-$ tienten erhalten durch ZorgDomein schneller einen Termin, Überweisungen in ein Krankenhaus erfolgen digital und unnötige Arztbesuche können so vermieden werden.

\subsection{Zusammenfassung und Fazit}

Die Probleme, die im Gesundheitswesen mit Schnittstellen einhergehen, insbesondere zwischen dem ambulanten und dem stationären Sektor, sind immer häufiger zentraler Gegenstand gesundheitspolitischer Diskussionen. Es wird dabei oftmals diskutiert, welche organisatorischen und strukturellen Rahmenbedingungen geändert werden müssen, um eine effektivere und effizientere Zusammenarbeit vor allem in der ambulanten und stationären Versorgung zu erreichen.

Der vorliegende Beitrag zeigt, dass diese Herausforderungen in anderen europäischen Ländern bereits konkreter angegangen wurden. Einige Länder haben in der jüngeren Vergangenheit entscheidende Reformen zur Re-Organisation der Gesundheitsversorgung durchgeführt, indem Zuständigkeiten zwischen den Sektoren verschoben wurden; einige Ansätze und dafür notwendige Voraussetzungen können durchaus auch im deutschen Kontext diskutiert werden.

Erstens zielen derzeitige Reformen in den untersuchten Ländern darauf, die Zuständigkeiten für die Steuerung und Planung von ambulanten und stationären Leistungen umzustrukturieren. Die Beispiele aus Norwegen, Finnland und Österreich haben gezeigt, dass nationale Gesundheitsreformen auf den Weg gebracht wurden, um eine gemeinsame integrative Versorgungsplanung sowie eine gemeinsame Steuerung und Finanzierung über die Sektorengrenzen hinweg zu schaffen und die Abhängigkeit vom Krankenhaussektor zu verringern. Die Reforminitiativen sind ein erster Schritt in eine fundamentale Umstrukturierung der Gesundheitssysteme. Insgesamt werden in fast allen europäischen Ländern zunehmend stationäre Leistungen ambulant durchgeführt.

Zweitens nutzen alle untersuchten Länder zunehmend das Prinzip des Gatekeeping durch Hausärzte bzw. Primärversorger, das eine verbesserte Kommunikation, Steuerung und Koordination zwischen ambulanter medizini- 
scher Versorgung und stationärer Behandlung ermöglicht. Insgesamt scheint es in den ausgewählten Ländern mittels der eingeführten Gatekeeper-Instrumente gelungen zu sein, die starre sektorale Trennung der unterschiedlichen Versorgungsbereiche zu überwinden.

Drittens sehen Reforminitiativen in z.B. Österreich, Finnland und Norwegen die Einführung multidisziplinärer Teams vor, um vor allem die Versorgung von Menschen mit komplexen Bedürfnissen über Schnittstellen hinweg besser zu koordinieren. Hierzu gehört auch die Einbindung von Gesundheitsfachberufen, die über eine reine Praxisassistenz hinausgehen. In Deutschland sind derartige Bestrebungen zur Stärkung der Primärversorgung zwar vorhanden (s. SVR 2009, 2014) jedoch bisher lediglich in Form von Modellprojekten umgesetzt. Eine Umsetzung und Überführung in die Regelversorgung fehlt bisher.

Viertens haben die meisten Länder mittels verschiedener Gesetzesinitiativen die Digitalisierung im Gesundheitswesen vorangetrieben, vor allem im Hinblick auf Interoperabilität, Patientenakten und Telemedizin. Einen entscheidenden Fortschritt in Bezug auf die Digitalisierung des Gesundheitssystems erzielten Dänemark und Estland mittels starker Steuerung, politischer Bereitschaft, frühzeitiger und verbindlicher Zielsetzung und der Schaffung von nationalen oder regionalen Rahmenbedingungen (Bertram et al. 2019; Thiel et al. 2018). Inhalte und Funktionen der elektronischen $\mathrm{Pa}$ tientenakte wurden von Anfang an klar definiert und Endanwender, also beispielsweise Patienten oder Leistungserbringer, wurden bei der Entwicklung aktiv mit einbezogen. Außerdem wurden technische sowie Interoperabilitätsstandards vorgegeben.

Derartige Initiativen könnten Teil zukünftiger bundesweiter Reformen werden und eine sektorenübergreifende Versorgung im deutschen Gesundheitswesen fördern.

\section{Literatur}

Albrecht M, Al-Abadi T, Czihal T, Mangiapane S (2020) Sektorenübergreifende Versorgung und Vergütung. In: Klauber J, Geraedts M, Friedrich J, Wasem J, Beivers A (Hrsg) Krankenhaus-Report 2020. Springer, Berlin, Heidelberg, S 243-261 https://doi.org/10. 1007/978-3-662-60487-8_13

Anderson GF (2011) The latest disease burden challenge: people with multiple chronic conditions. In: OECD (Hrsg) Health reform: meeting the challenge of ageing and multiple morbidities. OECD Publishing, Paris, S 15-35 https://doi.org/10.1787/9789264122314-en

Bachner F, Bobek J, Habimana K, Ladurner J, Lepuschutz L, Ostermann H, Rainer L, Schmidt AE, Zuba M, Quentin W, Winkelmann J (2019) Das österreichische Gesundheitssystem - Akteure, Daten, Analysen. Health Syst Transit 20(3):1-288. https://jasmin. goeg.at/434/13/Das\%20\%C3\%B6sterreichische $\% 20$ Gesundheitssystem_2019.pdf. Zugegriffen: 14. November 2020

Bertram N, Püschner F, Oliveira Gonçalves AS, Binder S, Amelung V (2019) Einführung einer elektronischen Patientenakte in Deutschland vor dem Hintergrund der internationalen Erfahrungen. In: Klauber J, Geraedts M, Friedrich J, Wasem J (Hrsg) Krankenhaus-Report 2019. Springer, Berlin, Heidelberg, S 3-16 https://doi. org/10.1007/978-3-662-58225-1_1

Czypionka T, Kraus M, Kronemann F (2015) Bezahlungssysteme in der Primärversorgung (Bd 2015). Wien. https://www.sozialversicherung.at/cdscontent/load? contentid $=10008.715455 \&$ version $=1477896840 . \mathrm{Zu}-$ gegriffen: 2. Sept. 2020

de Graaff R, Spee I (2018) Current developments in the Dutch healthcare market: Edition 2018. Seijgraaf Consultancy BV, Heemsteede. https://www. welfaretech.dk/media/6800/current-developmentsin-the-dutch-healthcare-market.pdf. Zugegriffen: 7. Sept. 2020

European Union (2017) State of health in the EU: companion report 2017. Publications office of the European Union, Luxembourg. https://ec.europa.eu/health/sites/ health/files/state/docs/2017_companion_en.pdf. Zugegriffen: 1. Sept. 2020

Friedrich-Ebert-Stiftung (FES) (2017) Positionspapier PATIENT FIRST! Für eine patientengerechte sektorenübergreifende Versorgung im deutschen Gesundheitswesen. 2017/03. http://library.fes.de/pdf-files/ wiso/13280.pdf. Zugegriffen: 2. Sept. 2020

Geissler A, Quentin W, Busse R (2016) Ambulante Leistungen von Krankenhäusern im europäischen Vergleich. In: Klauber J, Geraedts M, Friedrich J, Wasem J (Hrsg) Krankenhaus-Report 2016. Schwerpunkt: Ambulant im Krankenhaus. Schattauer, Stuttgart, S 29-41 
GKV-Spitzenverband (2017) Positionspapier des GKVSpitzenverbandes für die 19. Legislaturperiode 2017-2021. GKV-Spitzenverband, Berlin

IGES (2019) Internationaler Vergleich ambulanter Vergütung. Gutachten zu einem internationalen Vergleich der ambulanten ärztlichen Vergütung aus gesundheitsökonomischer Perspektive. Ergebnisbericht für das Bundesministerium für Gesundheit. https://www. bundesgesundheitsministerium.de/fileadmin/Dateien/ 5_Publikationen/Ministerium/Berichte/Gutachten_ IGES_-_internat_oek_Vergleich_bf.pdf. Zugegriffen: 2. Sept. 2020

Keskimäki I, Tynkkynen LK, Reissell E, Koivusalo M, Syrjä V, Vuorenkoski L, Rechel B, Karanikolos M (2019) Finland: health system review. Health Syst Transit 21(2):1-166

KOMV (2019) Empfehlungen für ein modernes Vergütungssystem in der ambulanten ärztlichen Versorgung (Bericht der Wissenschaftlichen Kommission für ein modernes Vergütungssystem - KOMV. Im Auftrag des Bundesministeriums für Gesundheit)

Kroneman M, Boerma W, van den Berg M, Groenewegen P, de Jong J, van Ginneken E (2016) The Netherlands: health system review. Health Syst Transit 18(2):1-239

Lai T, Habicht T, Kahur K, Reinap M, Kiivet R, Ginneken E (2013) Estonia: health system review. Health Syst Transit 15(6): 1-196

Leber W-D, Wasem J (2016) Ambulante Krankenhausleistungen - ein Überblick, eine Trendanalyse und einige ordnungspolitische Anmerkungen. In: Klauber J, Geraedts M, Friedrich J, Wasem J (Hrsg) Krankenhaus-Report 2016, Schwerpunkt: Ambulant im Krankenhaus. Schattauer, Stuttgart, S 3-28

Ministry of Social Affairs and Health, Finland (2020) https://stm.fi/en/primary-health-care. Zugegriffen: 10. Sept. 2020

Nagel E, Neukirch B, Schmid A, Schulte G (2017) Wege $\mathrm{zu}$ einer effektiven und effizienten Zusammenarbeit in der ambulanten und stationären Versorgung in Deutschland (Gutachten im Auftrag des Zentralinstitut für die kassenärztliche Versorgung in Bundesrepublik Deutschland (ZI))

Nair S, Oliver D, Cracknell A (2020) Meeting the needs of frail older patients. In: McKee M, Merkur S, Edwards $\mathrm{N}$, Nolte E (Hrsg) The changing role of the hospital in European health systems. European Observatory on Health Systems and Policies. Cambridge University Press, Cambridge, S 85-119 https://doi.org/10.1017/ 9781108855440.004

OECD/European Union (2018) Health at a glance: Europe 2018: state of health in the EU cycle. OECD publishing, Paris/European Union, Brussels. https:// doi.org/10.1787/health_glance_eur-2018-en. Zugegriffen: 30. Okt. 2020

OECD, European Observatory on Health Systems and Policies (2019a) Österreich: Länderprofil Gesundheit 2019, State of Health in the EU. OECD Publishing,
European Observatory on Health Systems and Policies, Paris, Brussels

OECD, European Observatory on Health Systems and Policies (2019b) Norway: country health profile 2019, state of health in the EU. OECD Publishing, European Observatory on Health Systems and Policies, Brussels, Brussels

OECD, European Observatory on Health Systems and Policies (2019c) The Netherlands: Country Health Profile 2019, State of Health in the EU. OECD Publishing, European Observatory on Health Systems and Policies, Paris, Brussels

Preusker U (2019) Reform des Gesundheitssystems in Finnland. In: Dormann F, Klauber J, Kuhlen R (Hrsg) Qualitätsmonitor 2019. Medizinisch Wissenschaftliche Verlagsgesellschaft, Berlin, S 3-18 https://doi. org/10.32745/9783954664344-1.1

Sachverständigenrat zur Begutachtung der Entwicklung im Gesundheitswesen (SVR) (2009) Koordination und Integration - Gesundheitsversorgung in einer Gesellschaft des längeren Lebens. Sondergutachten. Deutscher Bundestag Drucksache 16/13770

Sachverständigenrat zur Begutachtung der Entwicklung im Gesundheitswesen (SVR) (2012) Wettbewerb an der Schnittstelle zwischen ambulanter und stationärer Gesundheitsversorgung. Sondergutachten. Deutscher Bundestag Drucksache 17/10323

Sachverständigenrat zur Begutachtung der Entwicklung im Gesundheitswesen (SVR) (2014). Bedarfsgerechte Versorgung - Perspektiven für ländliche Regionen und ausgewählte Leistungsbereiche. Deutscher Bundestag Drucksache 18/1940

Sachverständigenrat zur Begutachtung der Entwicklung im Gesundheitswesen (SVR) (2018) Bedarfsgerechte Steuerung der Gesundheitsversorgung. Gutachten. Deutscher Bundestag Drucksache 19/3180

Schmidt AE, Bachner F, Rainer L, Zuba M, Bobek J, Lepuschütz L, Ostermann H, Winkelmann J, Quentin W (2018) Ambulatory care on the rise? Lessons from the Austrian health care reforms. Eurohealth 24(4):21-24

Schneider UK (2016) Einrichtungsübergreifende elektronische Patientenakten - zwischen Datenschutz und Gesundheitsschutz. Springer Vieweg, Wiesbaden https://doi.org/10.1007/978-3-658-11597-5

Sote-uudistus (2020) Health and social services reform. https://soteuudistus.fi/en/frontpage. Zugegriffen: 10. Sept. 2020

Sperre Saunes IS, Karanikolos M, Sagan A (2020a) Norway: health system review. Health Syst Transit 22(1): 1-163

Sperre Saunes IS, Sagan A, Karanikolos M (2020b) Norway's Healthcare Communities are set up to build bridges between hospitals and primary care. Eurohealth 26(1):29-33

Straub C, Bosch-Cleve B, Hölscher A, Walther A, Weineck S (2016) Versorgung patientenorientiert gestalten - sektorübergreifende Versorgung. In: Gesundheits- 
wesen aktuell. Beiträge und Analysen. Barmer GEK, S 14-35

Struckmann V, Quentin W, Busse R, van Ginneken E (2017) How to strengthen financing mechanisms to promote care for people with multimorbidity in Europe? Policy Brief 24. European Observatory on Health Systems and Policies, Brussels

Struijs JN, Til JT, Baan CA (2011) Experimenting with a bundled payment system for diabetes care in the $\mathrm{Ne}$ therlands: the first tangible effects. Int J Integr Care 2011(11):e139
Sundhed.dk (2020) https://www.sundhed.dk/borger/ service/om-sundheddk/ehealth-in-denmark/ background/. Zugegriffen: 25. Aug. 2020

Thiel R, Deimel L, Schmidtmann D, Piesche K, Hüsing T, Rennoch J, Stroetmann V, Kostera T (2018) Smart Health Systems: Auszug Portugal. Digitalisierungsstrategien im internationalen Vergleich $\mathrm{Bd}$. 1. Bertelsmann Stiftung, S 1-12

Wammes J, Stadhouders N, Westert G (2020) International health care system profiles, Netherlands. Commonwealth Fund. https://www.commonwealthfund. org/international-health-policy-center/countries/ netherlands. Zugegriffen: 4. Sept. 2020

Open Access Dieses Kapitel wird unter der Creative Commons Namensnennung 4.0 International Lizenz (http:// creativecommons.org/licenses/by/4.0/deed.de) veröffentlicht, welche die Nutzung, Vervielfältigung, Bearbeitung, Verbreitung und Wiedergabe in jeglichem Medium und Format erlaubt, sofern Sie den/die ursprünglichen Autor(en) und die Quelle ordnungsgemäß nennen, einen Link zur Creative Commons Lizenz beifügen und angeben, ob Änderungen vorgenommen wurden.

Die in diesem Kapitel enthaltenen Bilder und sonstiges Drittmaterial unterliegen ebenfalls der genannten Creative Commons Lizenz, sofern sich aus der Abbildungslegende nichts anderes ergibt. Sofern das betreffende Material nicht unter der genannten Creative Commons Lizenz steht und die betreffende Handlung nicht nach gesetzlichen Vorschriften erlaubt ist, ist für die oben aufgeführten Weiterverwendungen des Materials die Einwilligung des jeweiligen Rechteinhabers einzuholen. 